\title{
CAMBIOS DE COMPORTAMIENTOS SOCIOECONÓMICOS DE LA POBLACIÓN RURAL DE LA HUASTECA, $1985-2001^{1}$
}

\section{INTRODUCCIÓN}

El texto constituye un breve resúmen preliminar de la investigación encaminada hacia el análisis de los cambios de comportamientos socioeconómicos de los habitantes del campo Huasteca durante el período entre 1985 y 2001, realizada por un equipo de geógrafos polacos y mexicanos en julio del año 2001 en la parte Huasteca de los estados de Veracruz e Hidalgo (véase el mapa). ${ }^{2}$

El período 1985-2001 propuesto como la perspectiva temporal deriva de las experiencias del estudio sobre el terreno llevado a cabo en junio de 1985 en cerca de 40 localidades ubicadas en las tres zonas de la Región de las Huastecas, a saber la zona Litoral (entre Tamiahua, Naranjos y Cerro Azul), la zona Central (entre Huejutla, Platón Sánchez y Tempoal) y la zona Norte (entre Tamasopo, Ciudad Valles y Tamuín) concerniente a los patrones de estructuras rurales de Las Huastecas. ${ }^{2}$ En 1985 la recopilación de materiales empíricos a nivel local tuvo como propósito la definición de las características y problemas de las sociedades rurales, así como un intento del diagnóstico geográfico general de la situación regional (Dembicz, 1987, 11). ${ }^{3}$

Durante la investigación mencionada hemos tratado de recopilar tanto la información referente a la situación actual de aquel entonces, es decir la

1 Se entiende por La Huasteca la región sociocultural mexicana que abarca el norte del Edo de Veracruz, la parte oriental de San Luís Potosí, el sur de Tamaulipas y el noreste del Edo de Hidalgo. Según algunos autores el noroeste del Edo de Puebla y el noreste del de Querétaro también forman parte de la región. La región la habitan principalmente los mestizos con una proporción notoria de las comunidades indígenas nahua y teenek.

2 Los dos textos publicados a continuación el de Agata Zarzycka y el de Joanna Miętkiewska-Brynda conciernen a algunos aspectos de la misma investigación.

3 Fue realizado dentro del marco del Convenio de Colaboración Académica entre la Facultad de Geografía y Estudios Regionales de la Universidad de Varsovia y la entonces Escuela de Geografía de la Universidad Autónoma del Estado de México. Los resultados de la investigación fueron publicados en Varsovia, en polaco (Lokalne problemy wsi..., 1987) y en español (Estructuras..., 1986; Patrones..., 1987). 
correspondiente al año 1985, como la relacionada con la percepción de los cambios observados en la región entre 1975 y 1985, por la población rural así como por las autoridades municipales, algunas instituciones estatales y paraestatales, asociaciones de productores agropecuarios, etc.

El estudio previo, realizado en 1985, confirmó la tesis sobre una fuerte diversificación de la región tanto en su dimensión socio-cultural como en la económica y la consiguiente coexistencia de sistemas de la producción agropecuaria distintos con dinámicas y orientaciones diferentes. A pesar de la inexistencia de una política coherente del desarrollo rural y una fuerte atomización territorial de intereses económicos se ha podido constatar que entre 1975 y 1985 han mejorado las condiciones de vida de la población rural en relación con el acceso más fácil a los servicios sociales (educación y salud) y el desarrollo de la infraestructura técnica (transporte, electrificación, abastecimiento en agua potable). La situación de los proprietarios de algunos predios ha mejorado también gracias al crecimiento de la producción agropecuaria y mejores posibilidades de su comercialización (Czerny M., Dembicz A., Makowski J., Skoczek M., 1988). En la mayoría de los casos, los cambios observados han sido efecto de los programas del desarrollo rural integrado, implementados en México en la década de los setenta (tales como PIDER y COPLAMAR) y en principios de la década de los ochenta (SAM).

La investigación realizada en julio de $2001^{4}$ no constituyó una repetición de la anterior. El recorrido abarcó tan sólo 2 de las 3 zonas visitadas anteriormente: la zona Litoral (entre Tamiahua, Naranjos y Cerro Azul) y la zona Central (entre Huejutla, Platón Sánchez y Tempoal). El equipo visitó más de 20 localidades. ${ }^{5} \mathrm{El}$ interés principal consistía en observar los cambios en comportamientos socio-económicos de la población rural de la región -

4 Tal como en 1985 en la investigación tomaron parte geógrafos de la Facultad de Geografía y Estudios Regionales de la Universidad de Varsovia: dra Małgorzata Durydiwka, dr Joaquín R. González Martínez, dra Bogumiła Lisocka-Jaegermann, prof. Jerzy Makowski, mtra Joanna Miętkiewska-Brynda, mtra Agata Zarzycka, así como investigadores de la Universidad Autónoma del Estado de México: lic. Carlos Alberto Sara Gutiérrez, lic. Verónica Rico Urbina, lic. Alejandra González Becerril y dr Ricardo Rózga Luter.

5 Las localidades a visitar fueron seleccionadas en base de sus características socioeconómicas, detectadas en el estudio realizado en 1985: las zonas de Platón Sánchez, El Higo y Tempoál - por su estructura de la producción agropecuaria - el cultivo del tabaco en la primera, el cultivo de la caña de azúcar y por la ganadería en la segunda; la zona de Tamiahua - por sus atractivos turísticos debidos a su localización a las orillas de la Laguna de Tamiahua; la zona de Huejutla de Reyes - por el predominio de la población indígena involucrada tanto en la producción agropecuaria como en la artesanía tradicional. En total fueron visitadas 28 localidades agrupadas en los municipios siguientes: Tamiahua (Tamiahua, Tampache, Raya Obscura), Tuxpam (Palma Morelos, La Laja de Coloman), Cerro Azul (Cerro Azul, Juan Felipe), Chinampa (Chinampa) Tancoco (Zaragoza, Zacamixtle), Tepetzintla (Tepetzintla, El Humo, Tierra Blanca, Las Cañas), Chalma (Chalma), Platón Sánchez (Platón Sánchez, Corralilio), Tempoal de Sánchez (Tempoal de Sánchez), El Higo (El Higo, El Chote, Palmar Prieto), Huejutla (Huejutla, Tehuetlán, Macuxtepetla, Coacuilco, Ixcatlán, Santa Cruz), San Felipe Orizatlán (San Felipe Orizatlán). 


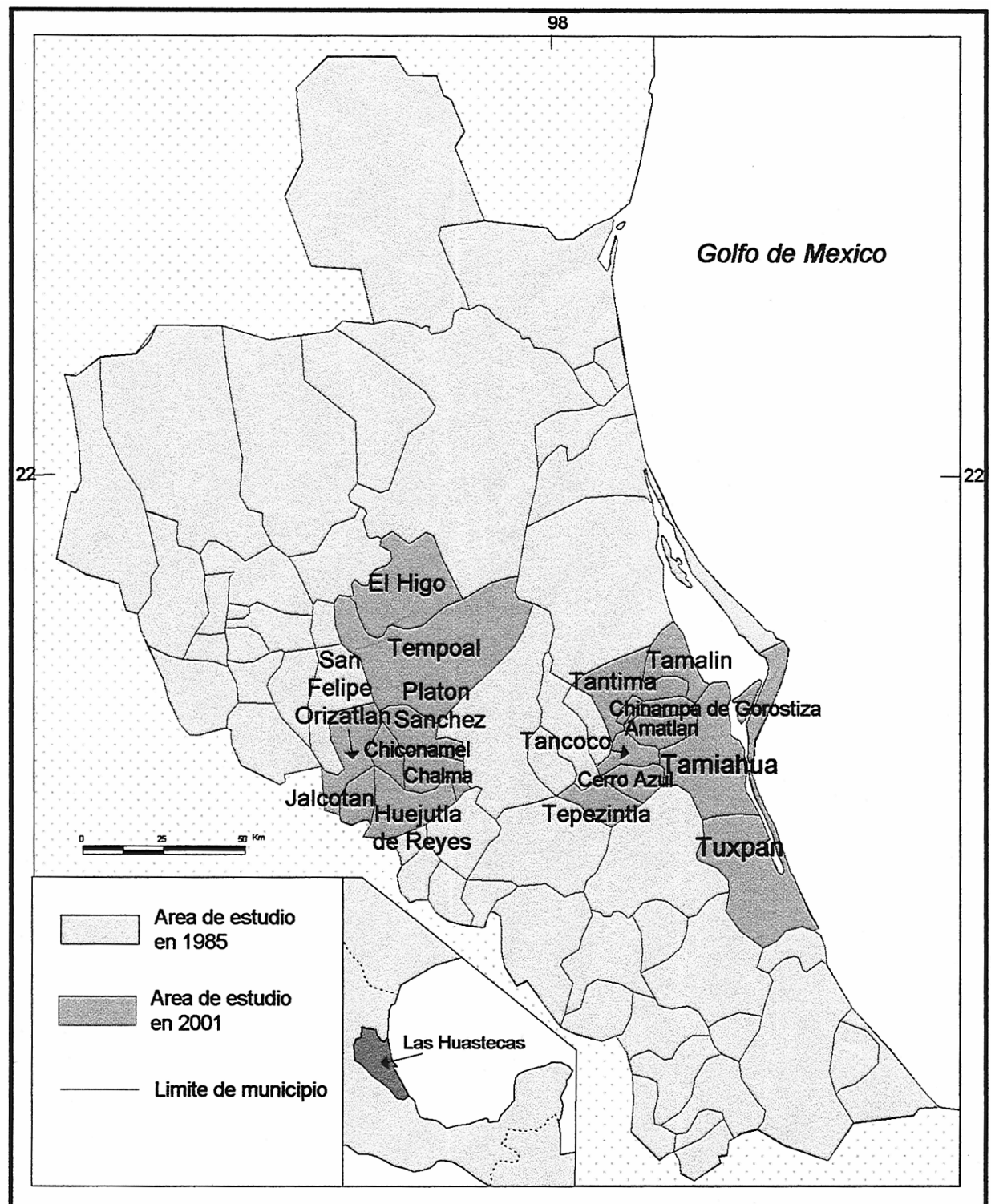

Fig. 1. La Huasteca. Localizacíon de la zona de estudio. Los municipios visitados.

tomando como el punto de partida la situación observada en 1985. Auque en el campo se aplicaron encuestas e inventarios similares a las del año 1985, el enfoque fue más bien cualitativo que cuántitativo. Se realizaron entrevistas abiertas con los representantes de las autoridades municipales y locales, así como con los agricultores, ganaderos, comerciantes. La visión de los cambios de la situación de la población rural presentada abajo, corresponde a la 
percepción de los mismos por los habitantes y por los investigadores quienes habían conocido La Huasteca hace 16 años. Los datos estadísticos oficiales correspondientes y accesibles hasta la fecha confirman dicha imagen.

\section{FACTORES DE CAMBIO DE LA SITUACIÓN DEL CAMPO EN LA HUASTECA}

Sin duda alguna en el período propuesto como el marco temporal de la investigación tanto en la sociedad como en la economía y en la vida política mexicanas han ocurrido cambios importantes. Entre los hechos y procesos más relevantes hay que mencionar la integración económica regional (el Tratado de Libre Comercio entre México, Estados Unidos de América y Canadá), así como otros elementos de las políticas neoliberales del Estado Mexicano: tales como, la supresión de los apoyos gubernamentales del campo a partir de 1989, la privatización y/o desincorporación de varias empresas agroindustriales paraestatales (Azúcar S.A., TABAMEX, INMECAFE), la reforma del artículo 27 de la Constitución de 1917 en 1992 y el consiguiente programa de regularización de tenencia de las tierras ejidales y comunitarias (Velasco Toro, 1993; Reyes Couturier et al., 1996). El marco nuevo de las realidades rurales mexicanas abarca también procesos de carácter político (el cambio del partido en poder y la activa presencia de distintos partidos políticos en el medio rural) y cultural (niveles de educación más altos, penetración de la cultura de masas más marcada, cambios en la vida religiosa).

Los estudios realizados por los investigadores mexicanos demuestran las transformaciones de comportamientos socioeconómicos de la población rural en varias regiones del país. ${ }^{6}$ Indican el agotamiento de las estrategias de sobrevivencia basadas unicamente, o casi unicamente en la agricultura de subsitencia - y la proletarización de la mano de obra agrícola (Peña, Morales, 1994, 149 -151), con el papel cada vez más importante de los ingresos de los migrantes rurales (Delgado, Figueroa, 1993, 6, citados por Mestries, 1994, 195).

Las opiniones citadas se inscriben en el amplio debate sobre el impacto de los procesos de globalización en la producción agropecuaria y en la vida de las sociedades rurales latinoamericanas. Aparte del aspecto económico del proceso, se van analizando las transformaciones sociales que éste implica. El concepto de los "campesinos nuevos", la aparición de nuevos actores sociales en el campo, movimientos campesinos y la transformación de las identidades sociales así como la necesidad de formulación de perspectivas teóricas alternativas parecen cambiar la idea del desarrollo rural en el continente americano (p.ej. Globalización, crisis..., 1998). En el caso de México dicho debate ha adquirido la dimensión del debate público en el verano de 2001.

6 (veáse, por ejemplo, los estudios sobre estados de Morelos y Zacatecas y las comarcas del Valle del Yaqui, Bajío guanajantense, La Laguna incluidos en el libro Campo y ciudad en una era de transición. Problemas, tendencias, desafíos, coordinado por Mario Bassols de la UAM-Unidad Iztapalapa (BASSOLS, 1994). 
Los periódicos informaban de una crisis agrícola aguda, de la dramática situación de la población rural y del peligro de una crisis social. ${ }^{7}$

En el caso de La Huasteca - las tendencias generales se inscriben dentro de un contexto regional específico. La Huasteca sigue siendo una región marginal (Ruvalcaba et al., 1993; Ruvalcaba, 1998). Como tal - no atrae la atención de inversionistas y muchos de los potenciales beneficios de la globalización económica - en su contexto resultan ilusorias. La decreciente actividad pesquera y camaronera de Tamiahua - dificilmente encuentra el mercado, en parte paralizada por el antiguo sistema de producción y ventas. La mitad de los años 80 marca el ocaso de la producción petrolera en Cerro Azul - lo que redujo considerablemente la oferta de los puestos de trabajo en la zona. En el mismo período se consolida el cuadro nuevo de tenencia de la tierra en las partes donde hubo conflictos e invasiones en los años 70-ta y 80-ta (principalmente en los alrededores de Huejutla y en menor grado en algunas zonas del norte de Veracruz), mientras que parecen manteniéndose las estructuras tradicionales del poder de los caciques locales. Con la activización de los partidos políticos de la zona las antiguas tensiones por la tierra se transforman en la competencia entre los grupos partidistas por los recursos, que según algunos de los estudiosos de la región permiten hacerla producir (Ramos Castro, 2000) Las organizaciones campesinas, surgidas a raíz de la lucha por la tierra toman parte en ella. La lucha entre los partidos políticos llega a nivel de las comunidades, y tal como lo indicaron algunos de nuestros respondientes, las asambleas se convierten en arenas de disputas entre fracciones políticas, problemas de cooperación con los regidores de otros partidos. El difícil tema de los conflictos por la tierra, parece trascender su marco temporal. La memoria de conflictos y de su carácter violento puede causar el apego más fuerte a la tierra - ganada con tanto esfuerzo - por poco productiva que sea. Por el otro - el hecho de que los protagonistas de los hechos pasados sigan siendo activos en la vida política local hace que el relativo equilibrio en la situación política local se considere vulnerable.

Las divisiones internas observadas a nivel local no se reducen al plano de las actividades de partidos políticos. El creciente número de miembros de grupos evangélicos en la zona tradicionalmente católica (y en especial en las comunidades), así como diferencias de opiniones acerca de las soluciones del problema de la tenecia de la tierra, y de su regularización manejada por el programa PROCEDE, competencia por el acceso a los programas de asistencia social (PROGRESA) constituyen otros factores de fragmentación de las comunidades que hace 16 años parecían mucho más cohesionadas.

Entre los factores de cambio observados a nivel nacional habría que recalcar aquellos que en la escala local adquirieron importancia especial. La desaparición de empresas paraestatales tuvo consecuencias graves para las zonas 'especializadas' desde hace tiempo en los cultivos comerciales. El cierre

7 véase p.ej. el texto de Armando Bartra en (Bartra, 2001) y los artículos publicados en la Jornada en julio y agosto de 2001 en la sección: 'Campo'. 
de TABAMEX ha afectado considerablemente todo el sector tabacalero de la zona del mpio de Platón Sánchez; la reorganización de la producción azucarera ha cambiado las condiciones de producción de la zona del Higo; la desaparición de INMECAFE ha reorganizado el sistema de comercialización del café en las comunidades de la zona de Huejutla.

\section{ESTRATEGIAS SOCIO-ECONÓMICAS DE LA POBLACIÓN RURAL}

Las técnicas de investigación aplicadas durante la estancia en la región del estudio permitieron observar la percepción de los procesos de cambios en el medio rural por sus habitantes. La mayoría de los entrevistados con ganas presentaban opiniones acerca de la situación actual del campo en su región, indicando que ésta se había transformado en el curso de los últimos años. Aunque notaban ciertas mejoras en su nivel de vida - entre los cuáles destacaban el mejor acceso a la educación y las facilidades del transporte local, por lo general indicaban que la 'vida es más difícil'. El nuevo contexto económico, político y social de la agricultura tradicional mexicana ha provocado la implementación de varias estrategías de sobrevivencia emprendidas por la población campesina, que han traido como resultado la diversificación de fuentes de ingreso de los habitantes del campo. Las informaciones obtenidas de las autoridades municipales, observaciones en las aldeas, cuestionarios que abarcaban su infraestructura formal e informal, entrevistas realizadas en las localidades más grandes durante los días de plaza, confirmaban los datos recopilados mediante las entrevistas con los agricultores y ganaderos, quienes presentaban las estrategias de sus propias familias y de las de vecinos de la misma localidad.

La mayoría de las personas entrevistadas han indicado que los ingresos provenientes de las actividades agrícolas tradicionales han disminuido considerablemente. La gente se limita al cultivo de la milpa tan sólo para las necesidades inmediatas de la familia o hasta renuncia del cultivo de maíz y frijol por completo. El programa de la regularización de tenencia de tierra en las comunidades indígenas y en los ejidos (PROCEDE) ya ha traido en consecuencia cambios considerables en algunas de las localidades. Hemos podido detectar tanto los casos, donde la tierra se vende únicamente a los locales que no disponen de parcelas propias o quienes quieran ampliarlas (el patrón predominante en las localidades indígenas), como los de aldeas cuyos habitantes vendieron tierra a quienes querían comprarla. Se hacían menciones de la crisis de los cultivos comerciales: del café, caña de azúcar y de cítricos. Entre las causas del fenómeno enumeradas por los informantes, aparecían tanto el cambio de las políticas estatales frente al campo, como los factores coyunturales del mercado (p.ej. la dramática caida de los precios del café), o los medioambientales.

Los entrevistados confirmaban la tendencia hacia la ganaderización del campo y la paulatina sustitución de explotaciones agrícolas por pastizales, observada cada vez más frecuentemente entre los propietarios 
de fincas pequeñas. Según las opiniones de los campesinos la cría de ganado ofrece más estabilidad, ya que se mantiene la demanda de carne en la región misma y en las grandes ciudades del pais, y siempre es posible adelantar o posponer la venta del animal, dependiendo de los precios en el mercado y de las necesidades de las familias. En algunas localidades es común arrendar la tierra por 2 hasta 5 años, a cambio de su transformación en pastos.

Entre los ganaderos que disponen de superficies más grandes hemos podido encontrar opiniones acerca de la crisis del sector - efecto de problemas de competitividad en el mercado estadounidense, así como las que postulaban cambios en las técnicas de producción y la transformación de leche en el lugar - como remedio en la situación actual en el sector. Es interesante el caso de los ganaderos de Las Cañas, visitados en 1985 cuando apenas iniciaban las transformaciones de la finca. Formando parte del Grupo Ganadero de Validación y Transferencia de Tecnología, gracias a la asistencia técnica brindada por el proyecto lograron introducir métodos nuevos del uso de pastizales y aumentar la productividad; también abrieron una pequeña planta de producción de quesos, lo que según ellos también forma parte del éxito de su empresa.

La producción artesanal de quesos para los clientes locales y para la venta en las ciudades grandes fue detectada también en las localidades donde predomina la propiedad ganadera más pequeña. El algunas comunidades de Huejutla (p.ej.Coacuilco) se produce miel. La transformación casera de carne, realizada a pequeña escala para satisfacer las necesidades de los vecinos del lugar parece constituir una fuente adicional de ingresos de algunos campesinos, tal como lo es la producción de pan en algunas de las casas que funcionan como panaderías informales especializadas en 'pan agrio' o 'pan dulce'. En Tepetzintla pudimos ver la producción casera de una especialidad de la cocina regional - el zacahuil - para la venta en el poblado y en los mercados de Cerro Azul. A veces las actividades mencionadas se hacen 'por encargo' - igual que una parte considerable de la producción artesanal tradicional - la elaboración de comales y cazuelas de barro, petates, escobas, muebles, blusas tradicionales bordadas a mano o máscaras usadas durante las celebraciones del Día de Muertos.Todos los productos enumerados se consideran objetos de uso y como tales se venden sólo en las localidades o en los mercados locales. A pesar de los valores estéticos no se promueve su venta ni a los clientes urbanos ni a turistas, quienes parecen no haber descubierto todavía valores de la Huasteca. La 'artesanía' - producida para la venta a clientes externos, la detectamos únicamente en los alrededores de Huejutla. Las empresas caseras de producción tienen como el fin proporcionar ingresos adicionales y por lo general, en las zonas rurales se combinan con agricultura o ganadería. En las zonas rurales existe el interés por desarrollar la pequeña agroindustria casera; los programas de microcréditos rurales (escasos según la mayoría de nuestros informantes) - conciernen, entre otros, a la producción de quesos, transformación de carne y las panaderías rurales. Muchos de los programas estatales o gubernamentales que tienen como el 
fin desarollar actividades nuevas en el medio rural están dirigidos principalmente hacia las mujeres. Tal es el caso del Programa Mujeres en Desarrollo del Edo de Veracruz, que abarca las zonas marginadas del estado y promueve la producción artesanal. Desgraciadamente algunas de las iniciativas que pude observar en el estado no traen efectos positivos, sea por falta de capital, sea por interpretaciones erróneas del carácter de las tradiciones artesanales femeninas en la región.

Los servicios informales y el comercio a pequeña escala constituyen otra fuente de ingreso adicional de las familias campesinas. En las aldeas funcionan tiendas minúsculas, las panaderías caseras mencionadas ya, cantinas informales, cervecerías. Tanto los productores, como los que se dedican al comercio como actividad principal venden en los tianguis en los centros comerciales de la región, siendo los de Cerro Azul, Huejutla, Tamiahua los más grandes. La movilidad comercial está relacionada con el desarrollo de servicios de transporte local. Existen rutas regulares entre casi todas las comunidades visitadas y centros más grandes. Algunos de los vehículos por lo general pertenecen a los habitantes locales. Entre los habitantes de las zonas rurales aparece también un grupo de comerciantes, quienes disponiendo de un vehículo propio realizan viajes por casi todo el país, vendiendo todo lo que tenga demanda. Sólamente algunos de ellos se ocupan de la distribución de los productos agrícolas locales (p.ej. quesos, miel) en los centros urbanos grandes (p.ej. Tampico, Ciudad de México, Reynosa).

Las migraciones laborales desde hace mucho tiempo han constituido un elemento fundamental de las estrategias de sobrevivencia de los habitantes del campo de La Huasteca. Dado que en el período 1985-2001 han cambiado considerablemente tanto sus direcciones como volúmenes, constituyen el tema de un artículo aparte (véase el texto de Joanna Miętkiewska-Brynda publicado en el presente tomo). Vale la pena subrayar su importancia para el desarrollo de las actividades productivas y comerciales descritas arriba. El trabajo fuera permite no sólo sobrevivir en las condiciones de una crisis agrícola - los recursos que proporciona pueden ser invertidos en una 'microempresa casera' - sea de producción o de servicios.

A pesar de la creciente movilidad espacial de la población rural de La Huasteca, observada en 2001 - en la región existe un evidente interés por soluciones agropecuarias alternativas para el campo. Los cultivos comerciales que se habían inserto ya en el panorama regional - el café en las comunidades de Huejutla, el tabaco en la zona de Platón Sánchez, han sufrido consecuencias de la desaparición de las empresas paraestatales mencionadas La INMECAFE y TABAMEX. Los productores afectados por dificultades en la comercialización y el bajo nivel de precios optan por el abandono de la producción, lo que ya es un hecho en el caso del tabaco. Lo mismo concierne a las plantaciones de naranja - dispersas por casi toda la región. El bajo nivel de precios y la falta de centros de acopio cercanos significaron pérdidas para los productores. La organización de la compra de caña de azúcar desanima también a los productores cañeros de los alrededores de EL Higo. 
Muchos prefieren arrendar sus tierras al ingenio que se ocupa tanto del cultivo como de la zafra. A pesar de las experiencias negativas con los cultivos comerciales mencionados - se observa una proliferación de proyectos e ideas - desarrollados tanto por las autoridades municipales como por grupos de campesinos - encaminados hacia la búsqueda de actividades agropecuarias 'nuevas' que pudieran constituir una alternativa tanto para la agricultura tradicional no - rentable, como para los cultivos comerciales en crisis.

Entre los proyectos en funcionamiento pudimos observar una de las pequeñas cooperativas de engorda de pollos que funcionan gracias al programa de microcréditos. La cooperativa de Juan Felipe, está formada por 6 mujeres. Constituye un ejemplo de iniciativa que proporciona ingresos a las familias pobres - algunas encabezadas por las mujeres, permite enriquecer la dieta de la población marginada y que a la vez cambia la situación de la mujer en una sociedad mestiza patriarcal. En algunas de las comunidades marginadas del municipio de Tempoál existen programas de engorda de cerdos y de la cría de ganado, basados en principios semejantes, en Chalma se brinda la asesoría técnica y legal para la cría de borregos y el cultivo de huertas familiares.

Los demás proyectos presentados estaban más bien en el etapa inicial. Entre los más interesantes destaca el de la reforestación contemplado por los habitantes de Tierra Blanca. Preparado con la ayuda gubernamental preve el cultivo de caoba, cedro, palo rosa. La madera puede ser aprovechada al cabo de 6-7 años. Los tabacaleros de Platón Sánchez organizados ya en una Sociedad de Solidaridad Social - una forma nueva de asociación de productores analizan las posibilidades de cultivo de papaya, chile, tomate, jícama y/o pastizales en vez de tabaco. Negocian con los compradores de la flor de jamaica y de nuevas variedades de tabaco (por el momento sin éxito), para obtener las garantías de compra - antes de invertir en cultivos nuevos. En el municipio de Tempoal y en Tehuetlán se propone desarrollar el cultivo de una de las variedades de palma - con destino a la producción de artesanías (petates, bolsas, sombreros y artefactos de camuflaje para la cacería de patos, solicitados por los canadienses.) En Ixcatlán algunos de los campesinos piensan cultivar vainilla, en Tempoál - jícama, 'ya que está constituye elemento de muchas dietas para adelgazar - muy de moda en los medios urbanos', etc. Es difícil estimar cuáles son las perspectivas de las iniciativas mencionadas. No cabe duda, sin embargo, que los habitantes de las zonas rurales se comportan como actores sociales activos, quienes no sólo encuentran soluciones inmediatas de sus problemas sino también buscan posibilidades de remediarlos a largo plazo, de crear bases económicas viables de la vida en el lugar.

En el caso de las zonas indígenas de la región de estudio, es imposible hablar de las estrategias de sobrevivencia de la población rural sin mencionar los programas del desarrollo federales dirigidos hacia las regiones marginadas de la República Mexicana. Aunque uno de ellos (PROCAMPO) tiene como el fin la modernización de la agricultura y el otro (PROGRESA) el mejoramiento 
de la alimentación y del estado de salud de niños y adolescentes, así como la elevación de niveles educación en las zonas marginadas, - ambos de hecho funcionan como programas de asistencia social. El primero permite cubrir los costos de insumos, mientras que el segundo significa pagos bimensuales a las familias con hijos que asisten a las escuelas. El pago se hace a la mujer. Depende de la cantidad de hijos (hasta 4 hijos por familia pueden contar con ayuda) y del nivel de educación al cual llegan (cuánto más alto - más grande el pago). La ayuda para niñas es más alta que para los varones. En el caso de las familias numerosas el pago puede alcanzar el volúmen considerable - hasta convertirse en la fuente principal de recursos financieros. Si el programa se mantiene en la forma actual puede constituir un factor de cambio decisivo en los municipios donde tiene más alcance. Se nos ha comentado que los niños que terminan la secundaria, por lo general se van a buscar trabajo fuera. migran. Las autoridades municipales de Huejutla estaban preocupadas por la tendencia creciente del índice de natalidad en las comunidades que se benefician del programa. Algunos de los campesinos indicaban que el PROGRESA divide las comunidades ya que no todas las familias reciben ayuda y a menudo los criterios de la selección de las participantes en el programa no están claras.

Resumiendo los resultados preliminares de las investigaciones relatadas en el presente texto hay que constatar que - la apertura comercial y la política estatal frente al sector agropecuario, la reforma del artículo 27 de la Constitución de 1917 han afectado el campo de La Huasteca provocando cambios en la producción agropecuaria desfavorables para la población rural. Si nos apegamos al modelo 'bimodal' de la transformación de la agricultura mexicana planteado por algunos investigadores, según el cual existe un sector agrícola moderno, integrado al mercado global, y el tradicional, excluido y condenado a la paulatina desaparición (Prud'homme et al., 1995) - la agricultura huasteca, salvo algunas excepciones pertenece al segundo. En la región no se observan inversiones grandes en la agricultura. La población campesina, que tal como lo ponen los periodistas 'sobra', 'ha dejado de ser funcional' bajo la política neoliberal actual, ${ }^{8}$ se ha visto obligada a implementar estrategias de sobrevivencia, que consisten principalmente en la diversificación de las fuentes de ingreso. Una parte de ellas se desarrollan en el ámbito local y regional, mientras que otras consisten en actividades que requieren movilidad espacial. Entre las últimas están las salidas hacia las explotaciones agrícolas modernas y hacia los centros de maquila en las zonas del norte del país. Dos tipos de estrategias interactuan entre sí. El campesino - es sin duda alguna, un sujeto activo, aún si consideramos que funciona y actúa dentro de un marco de exclusión, tal como lo plantea el modelo al que nos referimos. Concluir que es la exclusión el factor determinante de su situación sería erróneo. Al analizar las estrategias de las familias se nota que sus

8 Aviles, K., "Surgio con el neoliberalismo la crisis alimentaria" en la La Jornada 18, de Julio de 2001, p. 45. 
miembros entablan relaciones con el sistema económico dominante, insertándose en él y en muchos casos financiando gracias a la inclusión periódica o parcial su existencia dentro del marco de la exclusión. La multiplicidad de estrategias y situaciones observadas en el campo no permite sacar conclusiones simples, ya que los cambios del marco en el que se encuentra la agricultura huasteca son relativamente recientes. Los estudios ulteriores de las zonas rurales de La Huasteca son por lo tanto muy prometedores y tal vez ayuden a entender los procesos que ocurren en el campo latinoamericano a caballo de siglos.

\section{BIBLIOGRAFÍA}

Bas sols M., (coord.), 1994, Ciudad y campo en una era de transición. Problemas, tendencias, desafíos, UAM - Unidad Iztapalapa, México, D.F., 282 p.

Bartra A., 2001 "Un cafecito para el plan Puebla-Panamá", [en:] La Jornada 31 de julio de 2001, 12 p.

Czerny M., Dembicz A., Makowski J., Skoczek M., 1988, Rural improvement between 1975 and 1985 in Las Huastecas Region, Mexico, [en:] Hinderink J., Szulc-Dąbrowiecka E. (eds.), Successful Rural Development in Third World Countries, Nederlandse Geografische Studies 67, 155-165, Amsterdam/Utrecht.

Delgado R., Figueroa V., Hoffner M., Marquez U., 1993, Elementos para la transformación democrática del estado de Zacatecas, tesis de maestría en ciencia política, Universidad Autonoma de Zacatecas.

Dembicz A., 1987, Consideraciones metodológicas generales, [en:] Patrones, op. cit., 9-18.

Esparza Salinas R., Suárez B., Bonfil P., 1996, Las mujeres campesinas ante la Reforma al Capítulo 27 de la Constitución, GIMTRAP, México.

Estructuras rurales de Las Huastecas. Resultados preliminares de las investigaciones conjuntas polaco-mexicanas, 1986, Miscellanea Geographica, 297-319, Warszawa.

Globalizacion, Crisis y Desarrollo Rural en America Latina, 1998 (Asociación Latinoamericana de Sociología Rural. V Congreso Latinoamericano de Sociología Rural. Memoria de Sesones Plenarias) Colegio de Postgraduados. Universidad Autonoma de Chapingo, Texcox, México, 233 p.

De Gortari L., Ruvalcaba J., 1990, La Huasteca: vida y milagros. CIESAS, Cuadernos de la Casa Chata 173, México, 220 p.

Hernández Ferrer M., 2000, Ofrendas a Dhipak. Ritos agrícolas entre los teenek de San Luís Potosí. Tesis de licenciatura en etnohistoria, CIESAS.

Hoffmann O., Velazquez E. (coords.), 1994, Las Llanuras costeras de Veracruz, la lenta construcción de regiones, Universidad Veracruzana, ORSTOM, México, $340 \mathrm{p}$.

Lokalne problemy wsi regionu Las Huastecas w Meksyku, 1987, Polska lokalna i samorzad terytorialny $w$ warunkach reformy i rekonstrukcji gospodarki przestrzennej, WG i SR UW, IGP, Warszawa [en polaco].

Mestries F., 1994, Migración internacional y agricultura campesina en Zacatecas, [en:] Bassols M. (coord.), 1994, op. cit., 171-197.

Patrones de las estructuras rurales de Las Huastecas, 1987, Actas Latinoamericanas de Varsovia, t. 3, Warszawa, $256 \mathrm{p}$.

Peña de la S., Morales M., 1994, Productores y capitalismo agrario. El caso del maíz en Morelos, [en:] Bassols M. (coord.), op. cit. 1994, 147-170.

Ramos Castro E.E., Plata Vázquez J.L., 2000, Historia agraria y fraccionalismo político en la Huasteca hidalguense, Tesis de licenciatura en antropología social INAH CIESAS.

Reyes Couturier T., Alcalá Delgado E., Brunt Rivera L.M., Parcero López M., 1996, Campesinos, artículo 27 y el Estado mexicano, INAH, PyV, México. 
Romer M., 1995, Comunidad y migracion laboral en la Región Huasteca, Actas Latinoamericanas de Varsovia, t. 17, 125-139.

Ruvalcaba J., 1998, Nuevos aportes al. conocimiento de La Huasteca, CIESAS, México, $386 \mathrm{p}$.

Ruvalcaba J., Alcala G., (coords.), 1993, Huasteca, T. I, Espacio y tiempo. Mujer y trabajo, CIESAS SEP, México, $198 \mathrm{p}$.

Id., Huasteca, T. II, Prácticas agrícolas y medicina tradicional. Arte y sociedad, CIESAS SEP, México, $211 \mathrm{p}$.

Id., Huasteca, T. III, Movilizaciones campesinas, CIESAS SEP, México, $153 \mathrm{p}$.

Skoczek M., 1995, Migration and income distribution in rural areas of Latin America. The case of Mexico, Afryka, Azja, Ameryka Eacinska, t. 73, 71-79, Warszawa.

Vela s co Toro J., 1993, Política y legislación agraria en México. De la desamortización civil a la reforma campesina, CESLA UV, serie Estudios y Memorias, Warszawa, 165 p. 